\title{
Acute Fetal Anemia Diagnosed by Middle Cerebral Artery Doppler Velocimetry in Stage V Twin-Twin Transfusion Syndrome
}

\author{
Jennifer Salcedo, M.D., ${ }^{1}$ Esther Friedrich, M.D., ${ }^{2}$ Deborah A. Wing, M.D., ${ }^{2}$ \\ and Manuel Porto, M.D. ${ }^{2}$
}

In stage V twin-twin transfusion syndrome (TTTS), up to 50\% of surviving twins die or experience permanent disabilities, likely due to acute intertwin hemorrhage resulting in sudden severe anemia of the survivor. Although fetal middle cerebral artery (MCA) Doppler studies demonstrate strong correlation with fetal hemoglobin values, acute hemorrhagic events are more difficult to diagnose, and optimal timing of delivery of the survivor poses an obstetric dilemma. We report a case of newly diagnosed stage V TTTS at 28 weeks gestation, complicated by acute severe anemia diagnosed by significantly abnormal fetal MCA Doppler studies. The anemic twin was urgently delivered and is doing well without significant sequelae.

KEYWORDS: Fetal anemia, fetal demise, Doppler velocimetry, twin-twin transfusion syndrome

Acute inter-twin hemorrhage in stage $\mathrm{V}$ twintwin transfusion syndrome (TTTS) may be diagnosed using middle cerebral artery (MCA) Doppler velocimetry.

\section{CASE REPORT}

A 27-year-old gravida 3, para 1 at 23 weeks and 4 days of gestational age with a spontaneous monochorionic, diamniotic twin gestation presented to our institution for threatened preterm labor. Ultrasound evaluation upon admission revealed previously unknown stage I TTTS in otherwise anatomically normal female fetuses. The donor twin was noted to be a classic "stuck twin" without a measurable fluid pocket; the recipient showed severe polyhydramnios with a deepest vertical pocket (DVP) of $>13 \mathrm{~cm}$. Over the course of 9 days of hospitalization, three amnioreduction procedures were performed removing 900, 1400, and $1000 \mathrm{~mL}$ of amniotic fluid, respectively. The patient received magnesium sulfate tocolysis and betamethasone for fetal lung maturation. Her preterm labor resolved, and she was discharged home undelivered with close outpatient follow-up. Serial ultrasound examinations were performed and noted no progression of TTTS by Quintero staging criteria. However, the fluid discrepancy, particularly the amount of polyhydramnios, remained significant with 1.6- to $1.9-\mathrm{cm}$ pockets in the donor and $11-$ to $13-\mathrm{cm}$ pockets in the recipient twin. Five additional amnioreduction procedures were performed for maternal symptomatic relief
${ }^{1}$ Department of Obstetrics and Gynecology, ${ }^{2}$ Division of MaternalFetal Medicine, University of California, Irvine, Orange, California. Address for correspondence: Esther Friedrich, M.D., Assistant Professor (Volunteer), Division of Maternal-Fetal-Medicine, Department of Obstetrics and Gynecology, 101 The City Drive South, Bldg 56, Ste 800, Orange, CA 92868 (e-mail: efriedri@uci.edu; Esther. X.Friedrich@kp.org). No reprints available from author.
Am J Perinatol Rep 2011;1:95-98. Copyright (C) 2011 by Thieme Medical Publishers, Inc., 333 Seventh Avenue, New York, NY 10001, USA. Tel: +1 (212) 584-4662.

Received: January 30, 2011. Accepted after revision: June 20, 2011. Published online: August 3, 2011.

DOI: http://dx.doi.org/10.1055/s-0031-1285982.

ISSN 2157-6998. 
and improvement of the DVP in the donor to 6.5 to $6.8 \mathrm{~cm}$ was noted. At 28 weeks and 6 days of gestation, 2 days following a routine ultrasound evaluation that showed a DVP of $6.7 \mathrm{~cm}$ in the donor and $13 \mathrm{~cm}$ in the recipient twin as well as normal umbilical artery Doppler with a systolic to diastolic (S/D) ratio of 2.0 in the donor and 2.8 in the recipient, stage V TTTS was diagnosed with demise of the donor twin. The surviving recipient twin was noted to have a mildly increased umbilical artery S/D ratio of 4.9 with positive end-diastolic flow. Fetal heart rate tracing revealed minimal variability with intermittent variable and rare late decelerations. Repeat ultrasound evaluation after transfer to the labor and delivery unit included a fetal MCA peak systolic velocity (PSV) measurement and showed a marked increase to 2.27 multiples of the median (MoM) for gestational age.

Given the sudden progression and clinical symptoms of acute anemia in addition to a worsening fetal heart rate tracing, the decision was made to proceed with urgent repeat cesarean section. The demised donor twin A weighed $1206 \mathrm{~g}$. The surviving twin B weighed $1365 \mathrm{~g}$ with Apgar scores of 1 at 1 minute and 8 at 5 minutes (cord blood gases: arterial $\mathrm{pH} 7.31 ; \mathrm{pCO}_{2} 44.9$ mm Hg; $\mathrm{pO}_{2} 13.6 \mathrm{~mm} \mathrm{Hg} ; \mathrm{HCO}_{3} 22.0 \mathrm{mmol} / \mathrm{L}$; base excess -4; $\mathrm{O} 2$ saturation 14.2\%; venous $\mathrm{pH} 7.38$; $\mathrm{pCO}_{2} 39$ mm Hg; $\mathrm{pO}_{2} 30.4 \mathrm{~mm} \mathrm{Hg} ; \mathrm{HCO}_{3} 22.3 \mathrm{mmol} / \mathrm{L}$; base excess -3; $\mathrm{O}_{2}$ saturation $56.7 \%$ ). The newborn was pale and immediate blood analysis confirmed acute anemia with a hemoglobin of $8.1 \mathrm{~g} / \mathrm{dL}$ and a hematocrit of $27.8 \%$. She was intubated and received $15 \mathrm{~mL}$ of type $\mathrm{O}$-negative packed red blood cells. Maternal Kleihauer-Betke test was negative for indices of fetomaternal hemorrhage. Placental pathology demonstrated meconium-pigmented macrophages in the placental membranes, a velamentous cord insertion, a false knot, and generalized pallor. Multiple small artery-to-vein anastomoses were noted.

The infant's neonatal intensive care course was uncomplicated with the exception of a few typical complications of prematurity: mild hyperbilirubinemia and a short course of necrotizing enterocolitis were medically managed. At her 11-month follow-up, she met all developmental milestones and had not required additional specialist care.

\section{DISCUSSION}

TTTS complicates 10 to $15 \%$ of monochorionic twin pregnancies. ${ }^{1}$ The underlying pathophysiology involves unbalanced blood flow through placental vascular anastomoses. Stage V TTTS-defined as death of at least one twin-occurs in 32\% of such pregnancies. ${ }^{2}$ When not treated with laser ablation or another interventions, $\sim 50 \%$ of surviving twins die or experience permanent disabilities. ${ }^{3}$ Such disabilities are likely due to acute hemorrhage from surviving twin to deceased twin, resulting in severe anemia and ischemia-related damage, such as periventricular leukomalacia. ${ }^{4}$ These hemodynamic changes have been observed fetoscopically within 3 hours of initial twin demise..$^{5}$ Morbidity and mortality associated with significant preterm birth-occurring in $\sim 68 \%$ of these cases - are difficult to distinguish from the sequelae of acute ischemia. ${ }^{6}$ Given that the average

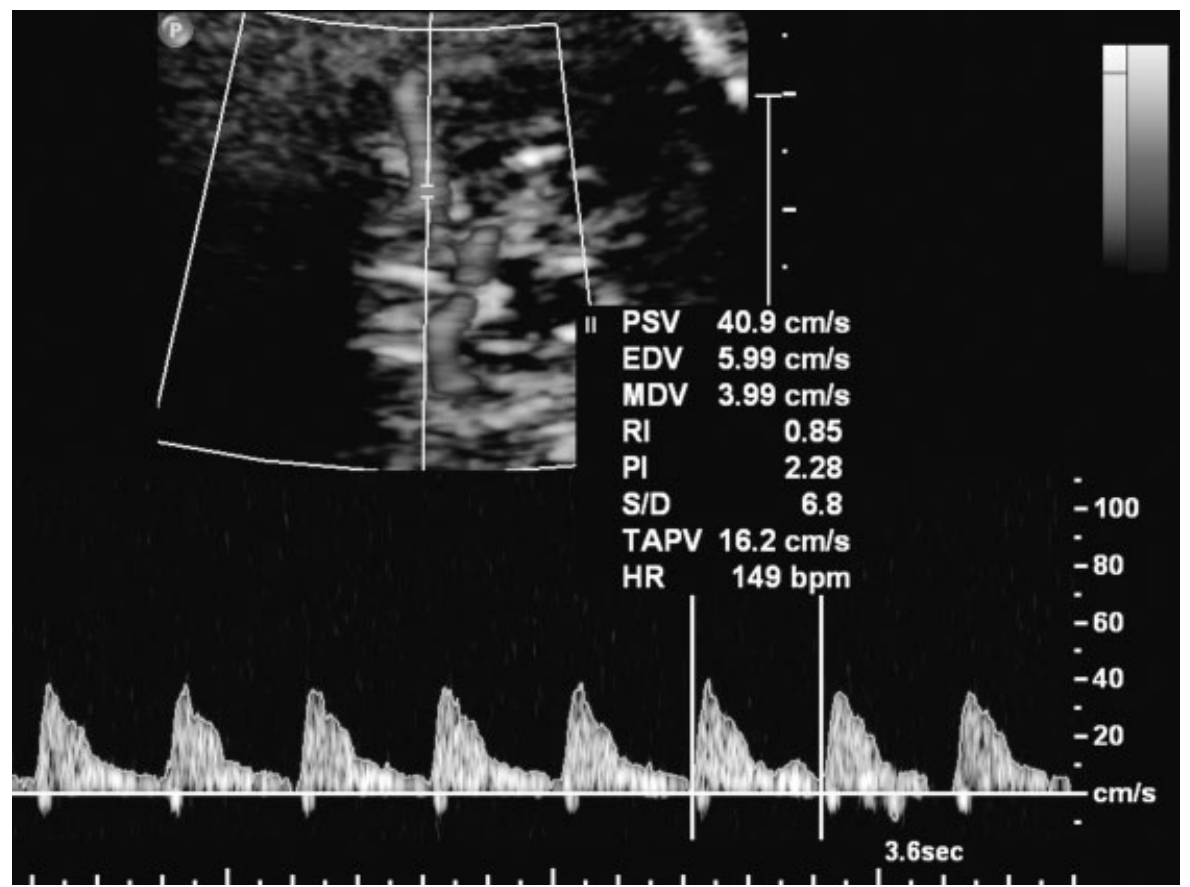

Figure 1 Example of a normal middle cerebral artery peak systolic velocity measurement of $40.9 \mathrm{~cm} / \mathrm{s}$ or 1.27 multiples of the median by Doppler ultrasound at 25 weeks gestational age. 


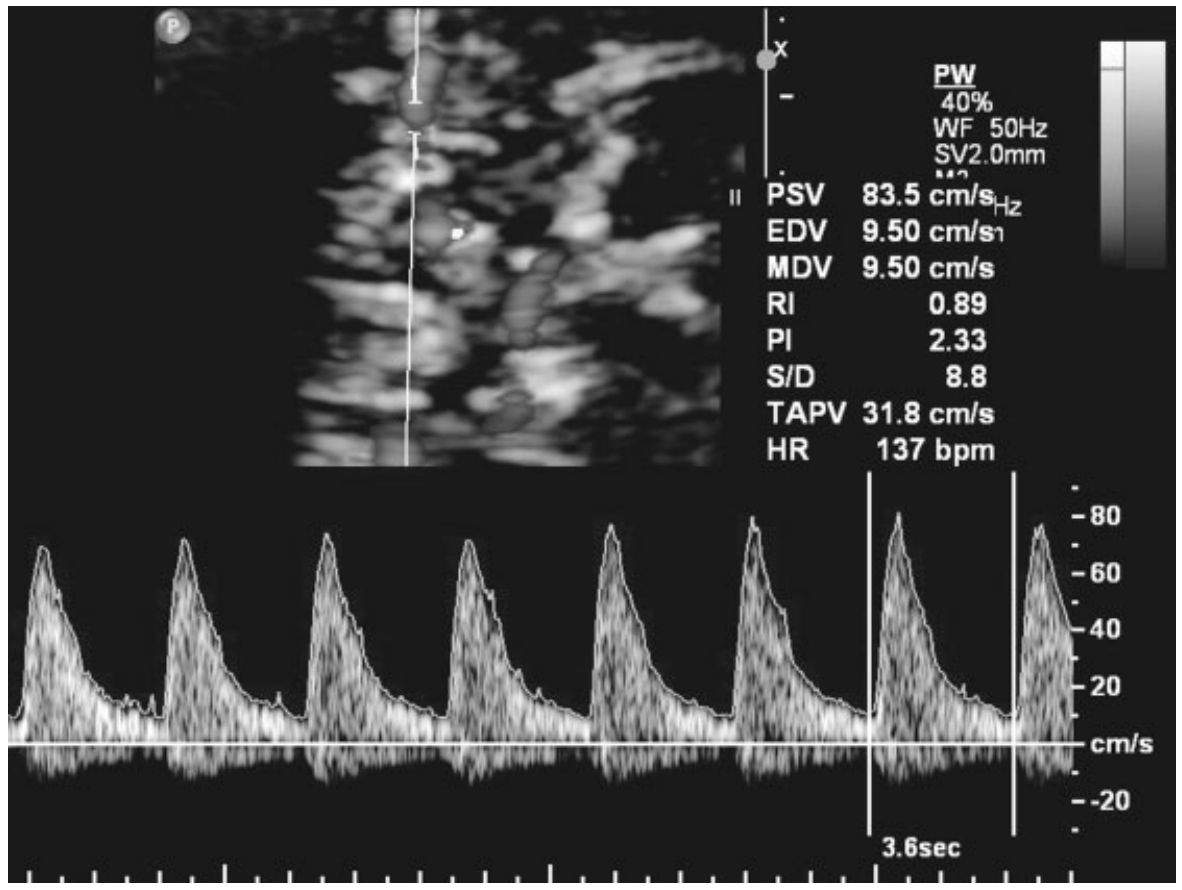

Figure 2 Example of an abnormal middle cerebral artery peak systolic velocity measurement of $83.5 \mathrm{~cm} / \mathrm{s}$ or $2.06 \mathrm{multiples}$ of the median by Doppler ultrasound at 30 weeks gestational age indicating fetal anemia.

gestational age at which death of the first twin occurs is 24 weeks, ${ }^{7}$ management of the surviving twin poses an obstetric dilemma. In our case, elevated fetal MCA Doppler PSV was used in the scenario of stage $\mathrm{V}$ TTTS to diagnose an acute intertwin hemorrhage. This ultimately led to delivery of a 28-week fetus due to concerns for impending fetal compromise and resulted in good long-term outcome.

TTTS in the lower stages is considered a chronic fetal condition that may be expectantly managed with careful observation to avoid complications of prematurity. ${ }^{1}$ Invasive procedures such as laser photocoagulation are not usually recommended for treatment in stage I TTTS. ${ }^{2}$ Sudden progression to a significantly higher stage is rare but associated with significant morbidity and mortality. ${ }^{1}$ In a very premature gestation as represented by our case, the decision to proceed with delivery rather than continue with expectant management or treatment by fetal transfusion must be carefully weighed. Because we could rely on a well-documented normal ultrasound just 2 days prior to the sudden deterioration, we were able to use the acute change manifested in the pathologically elevated fetal MCA Doppler measurement as an indicator of fetal compromise and make the decision to deliver urgently. We believe that despite the premature gestation, this rapid intervention and the subsequent neonatal transfusion led to the ultimately good outcome. Fetal MCA PSV of 1.5 MoM and greater has demonstrated $90 \%$ sensitivity and specificity for the prediction of moderate or severe fetal anemia ${ }^{5}$ (Figs. 1 and 2). The critically elevated value in this instance was likely due to reversal of blood flow through the anastomoses to the demised twin leading to anemia, as has been described previously in the literature. ${ }^{4} \mathrm{~A}$ few prior reports have discussed similar cases and avoided premature delivery by performing cordocentesis and rescue intrauterine transfusions. ${ }^{8}$ We argue that urgent delivery was likely preferable in our scenario, given the mixed reported results of such transfusions and the already compromised fetal heart rate tracing. Although we will not always have the luxury to be able to evaluate a twin pair with TTTS immediately at the time of demise of one twin to be able to intervene quickly and prevent demise or damage of the survivor, we believe that our case demonstrates how fetal MCA Doppler studies, even without corroboration by fetal blood sampling, can provide valuable information and may be integrated into the management of this rare but devastating complication. Physicians, sonographers, and parents of affected pregnancies should be aware of this possible outcome and have an emergent treatment plan in effect that should include immediate evaluation of the MCA Doppler PSV.

\section{REFERENCES}

1. Yamamoto M, Ville Y. Twin-to-twin transfusion syndrome: management options and outcomes. Clin Obstet Gynecol 2005;48:973-980 
2. Quarello E, Stirnemann J, Nassar M, et al. Outcome of anaemic monochorionic single survivors following early intrauterine rescue transfusion in cases of feto-fetal transfusion syndrome. BJOG 2008;115:595-601

3. Ohkuchi A, Minakami H, Shiraishi H, Suzuki I, Ohki T, Sato I. Intrauterine death of one twin, with rescue of the other, in twin-twin transfusion syndrome. Ultrasound Obstet Gynecol 2002;19:293-296

4. Senat MV, Loizeau S, Couderc S, Bernard JP, Ville Y. The value of middle cerebral artery peak systolic velocity in the diagnosis of fetal anemia after intrauterine death of one monochorionic twin. Am J Obstet Gynecol 2003;189:13201324

5. Quintero RA, Martínez JM, Bermúdez C, López J, Becerra C. Fetoscopic demonstration of perimortem feto-fetal hemor- rhage in twin-twin transfusion syndrome. Ultrasound Obstet Gynecol 2002;20:638-639

6. Ong SS, Zamora J, Khan KS, Kilby MD. Prognosis for the cotwin following single-twin death: a systematic review. BJOG 2006;113:992-998

7. Nicolini U, Pisoni MP, Cela E, Roberts A. Fetal blood sampling immediately before and within 24 hours of death in monochorionic twin pregnancies complicated by single intrauterine death. Am J Obstet Gynecol 1998;179(3 Pt 1):800803

8. Tanawattanacharoen S, Taylor MJ, Letsky EA, Cox PM, Cowan FM, Fisk NM. Intrauterine rescue transfusion in monochorionic multiple pregnancies with recent single intrauterine death. Prenat Diagn 2001;21:274278 